\title{
Magnetohydrodynamic Rotating Flow of a Fourth Grade Fluid Between Two Parallel Infinite Plates
}

\author{
M.A. Rana ${ }^{1 *}$, Akhlaq Ahmed ${ }^{2}$ and Rashid Qamar ${ }^{3}$ \\ ${ }^{1}$ Department of Basic Sciences, Riphah International University, Sector I-14, Islamabad, \\ ${ }^{2}$ Department of Mathematics, Quaid-i-Azam University, Islamabad, \\ ${ }^{3}$ Directorate of Management Information System, PAEC HQ, Islamabad,
}

Pakistan

\section{Introduction}

Mechanics of non-linear fluids present a special challenge to physicists, mathematician and engineers. The non-linearity can manifest itself in a variety of ways. Materials such as clay coatings and other suspensions, polymer melts, drilling muds, certain oils and greases, elastomers and many emulsions have been treated as non-Newtonian fluids. There is no single model which clearly exhibits all properties of non-Newtonian fluids and there has been much confusion over the classification of non-Newtonian fluids. However, nonNewtonian fluid may be classified as (1) fluids for which the shear stress depends only on the rate of shear; (2) fluids for which the relation between shear stress and shear rate depends on time; (3) the visco-elastoic fluids, which possess both elastic and viscous properties.

It is not possible to recommend a single constitutive equation which exhibits all properties of non-Newtonian fluids due to the great diversity in the physical structure of non-Newtonian fluids. For this reason, several non-Newtonian models or constitutive equations have been proposed and most of them are empirical or semi empirical. One of the simplest ways in which the visco-elastic fluids have been classified is the methodology given in $[1,2]$. They present constitutive relations for the stress tensor as a function of the symmetric part of the velocity gradient and its higher derivatives. Another class of models is the rate-type fluid models such as the Oldroyd model [3]. Although many constitutive equations have been suggested, many questions are still unsolved. Some of the continuum models do not give satisfactory results in accordance with the available experimental data. For this reason, in many practical applications, empirical or semi empirical equations have been used. A complete and thorough discussion of various models can be found in [4-7]. Various authors [8-12] investigated non-Newtonian fluids.

${ }^{*}$ Corresponding author 
The study of an electrically conducting fluid flows in channels under the action of a transversely applied magnetic field has important applications in many devices such as magnetohydrodynamic (MHD) pumps, aerodynamics heating, MHD power generators, accelerators, centrifugal separation of matter from fluid, flow meters, electrostatic precipitation, fluid droplets sprays, purification of crude oil, petroleum industries and polymer technology. Hartmann [13] first studied an incompressible viscous electrically conducting fluid under the action of a transverse magnetic field. Under different physical conditions it was considered by Sutton and Shermann [14], Hughes and Young [15], Cowling [16] and Pai [17]. Rajagopal and $\mathrm{Na}$ [18] studied the flow of a third grade fluid due to an oscillation of plate, Mollica and Rajagopal [19] examined secondary flows due to axial shearing of a third grade fluid between two eccentrically placed cylinders, Siddiqui and Kaloni [20] investigated plane flow of a third grade fluid. Rotating disk flows of conducting fluids have practical applications in many areas such as computer storage devices, lubrication, crystal growth processes, viscometry and rotating machinery. The effect of an external uniform magnetic field on the flow due to a rotating disk was studied [21-25], and eccentric rotation of disks was studied [26-29]. In many process of industries, the cooling of threads or sheets of some polymer materials is of great importance in the production line. Magneto convection plays an important role in various industrial applications including magnetic control of molten iron flow in the steel industry and liquid metal cooling in nuclear reactors. Palani and Abbas [30] investigated the combined effects of magnetohydrodynamic and radiation on free convection flow past an impulsively started isothermal vertical plate with Rosseland diffusion approximation, Farzaneh-Gord et al. [31] studied two-dimensional steady-state incompressible viscoelastic boundary layer magnetohydrodynamics flow and heat transfer over a stretching sheet in the presence of electric and magnetic fields. The highly non-linear momentum and heat transfer equations are solved analytically.

The MHD fluid flow as lubricant is of interest in industrial applications, because it prevents the unexpected variation of lubricant viscosity with temperature under certain extreme operating conditions. The MHD lubrication in an externally pressurized thrust bearing has been investigated both theoretically and experimentally by Maki et al. [32]. Hughes and Elco [33] and Kuzma et al. [34] have investigated the effects of a magnetic field in lubrication. These authors had neglected the inertial terms in the Navier-Stokes equations. Hamza [35] considered the squeezing flow between two discs in the presence of a magnetic field. The problem of squeezing flow between rotating discs has been studied by Hamza [36] and Bhattacharyya and Pal [37]. Considering two-dimensional unsteady MHD flow of a viscous fluid between two moving parallel plates, Sweet et al. [38] have shown that the flow is strongly influenced by the strength of the magnetic field and the density of the fluid. Abbas et al. [39] have investigated the unsteady MHD boundary layer flow and heat transfer in an incompressible rotating viscous fluid over a stretching continuous sheet. The resulting system of partial differential equations is solved numerically using Keller-box method. Turkylimazoglu [40] has analyzed the MHD time-dependent von Karman swirling electrically conducting viscous fluid flow having a temperature-dependent viscosity due to a rotating disk impulsively set into motion. 
Hayat et al. [41] have considered the unsteady rotating MHD flow of an incompressible second grade fluid in a porous half space. The flow is induced by a suddenly moved plate in its own plane. Both the fluid and plate rotate in unison with the same angular velocity. Assuming the velocity field of the form $\mathbf{V}=[u(z, t), v(z, t), w(z, t)]$, analytical solutions are presented using Fourier sine transforms and it is shown that with an increase in MHD parameter the real and imaginary parts of velocity as well as the boundary layer thickness decreases.

The classical theories of continuum mechanics are inadequate to explicate the microscopic manifestations of microscopic events. The fluids with microstructure belonging to a class of fluid with non-symmetrical stress tensor referred to as polar fluids are called Micropolar fluids. Physically they represent fluids consisting of randomly oriented particles suspended in a viscous medium. Eringen [42] presented the earliest formulation of a general theory of fluid microcontinua taking into account the inertial characteristics of the substructure particles which are allowed to undergo rotation in 1964. This theory has been extended by Eringen [43] to take into account thermal effects. The theory of micropolar fluids and its extension thermomicropolar fluids [44] may form suitable nonNewtonian fluid models which can be used to explain the flow of colloidal fluids, polymeric suspensions, liquid crystals, animal blood, etc. Eldabe et al. [45] have discussed the problem of heat transfer to MHD flow of a micropolar fluid from a stretching sheet with suction and blowing through a porous medium. The numerical results indicate that the velocity and the angular velocity increase as the permeability parameter increases but they decrease as the magnetic field increases. On the other hand, the temperature decreases as the permeability parameter increases but it increases as the magnetic field increases.

The study of laminar boundary layer flow of non-Newtonian fluids over continuous moving surfaces is very important because of its practical importance in a number of engineering processes. For example, cooling of an infinite metallic plate in a cooling bath, the boundary layer along a liquid film in condensation processes, aerodynamic extrusion of plastic sheets and a polymer sheet or filament extruded continuously from a dye. Furthermore, it has several practical applications in the field of metallurgy and chemical engineering such as material manufactured by extrusion process and heattreated materials traveling between a feed roll and a wind-up roll or on conveyor belt possess, the feature of a moving continuous surface. Also, glass blowing, continuous casting, and spinning of fibers involve the flow due to a stretching surface. Sarpakaya [46] studied the MHD flow of a non-Newtonian fluid, Char [47] studied the MHD flow of a viscoelastic fluid over a stretching sheet by considering the thermal diffusion in the energy equation. However, the effects of thermal radiation on the viscoelastic boundary layer flow and heat transfer can be quite significant at high operating temperatures. In view of this, Raptis [48], Raptis and Perdikis [49] and Raptis et al. [50] studied the viscoelastic flow and heat transfer over a flat plate with constant suction, thermal radiation and viscous dissipation. Recently, the effects of viscous dissipation, radiation, in presence of temperature dependent heat sources/sinks on heat transfer characteristics of a viscoelastic fluid is considered by Siddheshwar and Mahabaleswar [51]. Khan [52] extended the problem by including the effects of suction/injection, heat source/sink and radiation effects. Abel et al. [53] investigated the effects of viscous dissipation and non- 
uniform heat source on viscoelastic boundary layer flow over a linear stretching sheet. Abel and Nandeppanavar [54] studied the effect of non-uniform heat source/sink on MHD viscoelastic boundary layer flow, further Nandeppanavar et al. [55] studied the effects of elastic deformation and non-uniform heat source on viscoelastic boundary layer flow. Motivated by these studies, Mahantesh et al. [56] extended the results of researchers [53,54,55] for MHD viscoelastic boundary layer flow with combined effects of viscous dissipation, thermal radiation and non-uniform heat source which was ignored by $[53,54,55]$. Furthermore, they analyzed the effects of radiation, viscous dissipation, viscoelasticity, magnetic field on the heat transfer characteristics in the presence of nonuniform heat source with variable PST and PHF temperature boundary conditions. Kayvan Sadeghy et al. [57] have investigated theoretically the applicability of magnetic fields for controlling hydrodynamic separation in Jeffrey-Hamel flows of viscoelastic fluids. It is shown that for viscoelastic fluids, it is possible to delay flow separation in a diverging channel provided that the magnetic field is sufficiently strong. It is also shown that the effect of magnetic field on flow separation becomes more pronounced the higher the fluid's elasticity.

In the present paper we have modeled the unsteady flow equations of a fourth grade fluid bounded between two non-conducting rigid plates in a rotating frame of reference with imposed uniform transverse magnetic field. It is interesting to note that we are able to couple the equations arising for the velocity field. The steady rotating flow of the nonNewtonian fluid subject to a uniform transverse magnetic field is studied. The non-linear differential equations resulting from the balance of momentum and mass are solved numerically. The effects of exerted magnetic field, Ekman number and material parameter on the velocity distribution are presented graphically. The results for Newtonian and nonNewtonian fluids are compared.

\section{Mathematical model of the problem}

We introduce a Cartesian coordinate system with z-axis normal to the plane of the parallel plates. The plates are located at $z=0$ and $z=L$ and the plates and the fluid bounded between them are in a rigid body rotation with constant angular velocity $\Omega$ about the zaxis. The fluid is electrically conducting and assumed to be permeated by an imposed magnetic field $B_{0}$ perpendicular to the parallel plates. The disturbance in the fluid is produced by small amplitude non-torsional oscillations of the lower plate. For the present model we take the velocity field of the form.

$$
\mathbf{V}=[u(z, t), v(z, t), 0],
$$

where $u$ and $v$ are the $\mathrm{x}$ and $\mathrm{y}$ components of the velocity field. The Cauchy stress tensor for the fourth grade fluid can be obtained by the model introduced by Coleman and Noll [58]

$$
\mathbf{T}=-p \mathbf{I}+\sum_{j=1}^{n} \mathbf{S}_{j}
$$

For the fourth grade fluid we have $n=4$ and the first four tensors $S_{j}$ are given by 


$$
\begin{gathered}
\mathbf{S}_{1}=\mu \mathbf{A}_{1}, \\
\mathbf{S}_{2}=\alpha_{1} \mathbf{A}_{2}+\alpha_{2} \mathbf{A}_{1}^{2}, \\
\mathbf{S}_{3}=\beta_{1} \mathbf{A}_{3}+\beta_{2}\left(\mathbf{A}_{2} \mathbf{A}_{1}+\mathbf{A}_{1} \mathbf{A}_{2}\right)+\beta_{3}\left(\operatorname{tr} \mathbf{A}_{1}^{2}\right) \mathbf{A}_{1}, \\
\mathbf{S}_{4}=\gamma_{1} \mathbf{A}_{4}+\gamma_{2}\left(\mathbf{A}_{3} \mathbf{A}_{1}+\mathbf{A}_{1} \mathbf{A}_{3}\right)+\gamma_{3} \mathbf{A}_{2}^{2} \\
+\gamma_{4}\left(\mathbf{A}_{2} \mathbf{A}_{1}^{2}+\mathbf{A}_{1}^{2} \mathbf{A}_{2}\right)+\gamma_{5}\left(\operatorname{tr} \mathbf{A}_{2}\right) \mathbf{A}_{2}+\gamma_{6}\left(\operatorname{tr} \mathbf{A}_{2}\right) \mathbf{A}_{1}^{2} \\
+\left\{\gamma_{7}\left(\operatorname{tr} \mathbf{A}_{3}\right)+\gamma_{8}\left(\operatorname{tr} \mathbf{A}_{2} \mathbf{A}_{1}\right)\right\} \mathbf{A}_{1},
\end{gathered}
$$

where $\mu$ is the co-efficient of shear viscosity; and

$$
\alpha_{i}(i=1,2), \beta_{j}(j=1,2,3), \gamma_{k}(k=1,2, \ldots, 8)
$$

are material constants. The Rivlin- Ericken tensors $\mathbf{A}_{n}$ are defined by the recursion relation

$$
\begin{gathered}
\mathbf{A}_{n}=\frac{d \mathbf{A}_{n-1}}{d t}+\mathbf{A}_{n-1}(\operatorname{grad} \mathbf{V})+(\operatorname{grad} \mathbf{V})^{T} \mathbf{A}_{n-1}, n>1, \\
\mathbf{A}_{1}=(\operatorname{grad} \mathbf{V})+(\operatorname{grad} \mathbf{V})^{T},
\end{gathered}
$$

where

$$
\frac{d}{d t}(.)=\left(\frac{\partial}{\partial t}+\mathbf{V} \cdot \nabla\right)(.)
$$

When $\gamma_{k}=0(k=1,2, \ldots, 8)$, the fourth grade model reduces to third grade model, when $\beta_{j}=0(j=1,2,3)$ and $\gamma_{k}=0(k=1,2, \ldots, 8)$ then above model reduces to second grade model and if $\alpha_{i}=0(i=1,2), \beta_{j}=0(j=1,2,3), \gamma_{k}=0(k=1,2, \ldots, 8)$ the flow model reduces to classical Navier-Stokes viscous fluid model.

The hydromagnetic flow is generated in the uniformly rotating fluid by small amplitude non-torsional oscillations of the plate located at $z=0$. With the Cartesian coordinate system $O_{x y z}$ the unsteady motion of the incompressible fourth grade conducting fluid in the presence of magnetic field $\mathbf{B}$ is governed by the law of balance of linear momentum and balance of mass i.e.

$$
\begin{gathered}
\frac{d \mathbf{V}}{d t}+2(\boldsymbol{\Omega} \times \mathbf{V})+\boldsymbol{\Omega} \times(\boldsymbol{\Omega} \times \mathbf{r})=\frac{1}{\rho} \operatorname{div} \mathbf{T}+\frac{1}{\rho}(\mathbf{J} \times \mathbf{B}), \\
\operatorname{div} \mathbf{V}=0,
\end{gathered}
$$


where $\rho$ is the density, $\mathbf{J}$ is the current density and $\mathbf{B}\left(=\mathbf{B}_{0}+\mathbf{b}, \mathbf{b}\right.$ being the induced magnetic field) is the total magnetic field.

In the absence of displacement currents, the Maxwell equations and the generalized Ohm's law can be written as

$$
\begin{gathered}
\nabla \cdot \mathbf{B}=0, \nabla \times \mathbf{B}=\mu_{m} \mathbf{J}, \nabla \times \mathbf{E}=-\frac{\partial \mathbf{B}}{\partial t}, \\
\mathbf{J}=\sigma(\mathbf{E}+\nabla \times \mathbf{B}),
\end{gathered}
$$

where $\mu_{m}$ is the magnetic permeability, $\mathbf{E}$ is the electric field and $\sigma$ is the electrical conductivity of the fluid.

The magnetic Reynolds number is assumed to be very small so that the induced magnetic field is negligible [14]. This assumption is reasonable for the flow of liquid metals, e.g. mercury or liquid sodium (which are electrically conducting under laboratory conditions). The electron-atom collision frequency is assumed to be relatively high so that the Hall effect can be included [14]. The Lorentz force per unit volume is given by

$$
\mathbf{J} \times \mathbf{B}=-\sigma B_{0}^{2} \mathbf{V}
$$

For the velocity field defined in Eq. (1), the equation of continuity (11) is identically satisfied and Eq. (10) in component form can be written as

$$
\begin{aligned}
& \frac{\partial u}{\partial t}-2 \Omega v-\Omega^{2} x=-\frac{1}{\rho} \frac{\partial p}{\partial x}+\frac{\mu}{\rho} \frac{\partial^{2} u}{\partial z^{2}}+\frac{\alpha_{1}}{\rho} \frac{\partial^{3} u}{\partial z^{2} \partial t}+\frac{\beta_{1}}{\rho} \frac{\partial^{4} u}{\partial z^{2} \partial t^{2}} \\
& +\frac{2\left(\beta_{2}+\beta_{3}\right)}{\rho} \frac{\partial}{\partial z}\left[2 \frac{\partial u}{\partial z}\left\{\left(\frac{\partial u}{\partial z}\right)^{2}+\left(\frac{\partial v}{\partial z}\right)^{2}\right\}\right] \\
& +\frac{\gamma_{2}}{\rho} \frac{\partial}{\partial z}\left[\frac{\partial u}{\partial z}\left\{\begin{array}{l}
2 \frac{\partial}{\partial t}\left(\left(\frac{\partial u}{\partial z}\right)^{2}+\left(\frac{\partial v}{\partial z}\right)^{2}\right) \\
\left.+2 \frac{\partial u}{\partial z} \frac{\partial^{2} u}{\partial z \partial t}+2 \frac{\partial v}{\partial z} \frac{\partial^{2} v}{\partial z \partial t}\right)
\end{array}\right\}\right] \\
& +\frac{\left(\gamma_{3}+\gamma_{5}\right)}{\rho} \frac{\partial}{\partial z}\left[2 \frac{\partial^{2} u}{\partial z \partial t}\left\{\left(\frac{\partial u}{\partial z}\right)^{2}+\left(\frac{\partial v}{\partial z}\right)^{2}\right\}\right] \\
& +\frac{\partial}{\partial z}\left[\frac{\partial u}{\partial z}\left\{\begin{array}{c}
2 \frac{\gamma_{7}}{\rho} \frac{\partial}{\partial t}\left(\left(\frac{\partial u}{\partial z}\right)^{2}+\left(\frac{\partial v}{\partial z}\right)^{2}\right) \\
+\frac{\left(\gamma_{7}+\gamma_{8}\right)}{\rho}\left(\frac{\partial u}{\partial z} \frac{\partial^{2} u}{\partial z \partial t}+\frac{\partial v}{\partial z} \frac{\partial^{2} v}{\partial z \partial t}\right)
\end{array}\right\}\right]+\frac{\gamma_{1}}{\rho} \frac{\partial^{5} u}{\partial z^{2} \partial t^{3}}-\frac{\sigma}{\rho} B_{0}^{2} u,
\end{aligned}
$$




$$
\begin{aligned}
\frac{\partial v}{\partial t}+2 \Omega u-\Omega^{2} y & =-\frac{1}{\rho} \frac{\partial p}{\partial y}+\frac{\mu}{\rho} \frac{\partial^{2} v}{\partial z^{2}}+\frac{\alpha_{1}}{\rho} \frac{\partial^{3} v}{\partial z^{2} \partial t}+\frac{\beta_{1}}{\rho} \frac{\partial^{4} v}{\partial z^{2} \partial t^{2}} \\
& +\frac{2\left(\beta_{2}+\beta_{3}\right)}{\rho} \frac{\partial}{\partial z}\left[2 \frac{\partial v}{\partial z}\left\{\left(\frac{\partial u}{\partial z}\right)^{2}+\left(\frac{\partial v}{\partial z}\right)^{2}\right\}\right] \\
& +\frac{\gamma_{2}}{\rho} \frac{\partial}{\partial z}\left[\frac{\partial v}{\partial z}\left\{2 \frac{\partial}{\partial t}\left(\left(\frac{\partial u}{\partial z}\right)^{2}+\left(\frac{\partial v}{\partial z}\right)^{2}\right)\right\}\right] \\
& \left.\left.\left.+\frac{\left(\gamma_{3}+\gamma_{5}\right)}{\rho} \frac{\partial}{\partial z}\left[2 \frac{\partial u}{\partial z} \frac{\partial^{2} u}{\partial z \partial t}+2 \frac{\partial v}{\partial z} \frac{\partial^{2} v}{\partial z \partial t}\right)\right]\left(\frac{\partial u}{\partial z}\right)^{2}+\left(\frac{\partial v}{\partial z}\right)^{2}\right\}\right] \\
& +\frac{\partial}{\partial z}\left[\frac{\partial v}{\partial z}\left\{\begin{array}{l}
\left.2 \frac{\gamma_{7}}{\rho} \frac{\partial}{\partial t}\left(\left(\frac{\partial u}{\partial z}\right)^{2}+\left(\frac{\partial v}{\partial z}\right)^{2}\right)\right] \\
\left.\left.+\frac{\left(\gamma_{7}+\gamma_{8}\right.}{\rho}\left(\frac{\partial u}{\partial z} \frac{\partial^{2} u}{\partial z \partial t}+\frac{\partial v}{\partial z} \frac{\partial^{2} v}{\partial z \partial t}\right)\right]\right]+\frac{\gamma_{1}}{\rho} \frac{\partial^{5} v}{\partial z^{2} \partial t^{3}}-\frac{\sigma}{\rho} B_{0}^{2} v
\end{array}\right]\right.
\end{aligned}
$$

$$
\begin{aligned}
& 0=-\frac{1}{\rho} \frac{\partial p}{\partial z}+\frac{\left(2 \alpha_{1}+\alpha_{2}\right)}{\rho} \frac{\partial}{\partial z}\left\{\left(\frac{\partial u}{\partial z}\right)^{2}+\left(\frac{\partial v}{\partial z}\right)^{2}\right\}+\frac{\beta_{1}}{\rho} \frac{\partial}{\partial z}\left\{\begin{array}{l}
2 \frac{\partial}{\partial t}\left(\left(\frac{\partial u}{\partial z}\right)^{2}+\left(\frac{\partial v}{\partial z}\right)^{2}\right) \\
+2 \frac{\partial u}{\partial z} \frac{\partial^{2} u}{\partial z \partial t}+2 \frac{\partial v}{\partial z} \frac{\partial^{2} v}{\partial z \partial t}
\end{array}\right\} \\
& \left(2 \frac{\partial^{2}}{\partial t^{2}}\left(\left(\frac{\partial u}{\partial z}\right)^{2}+\left(\frac{\partial v}{\partial z}\right)^{2}\right)\right. \\
& +\frac{\beta_{2}}{\rho} \frac{\partial}{\partial z}\left\{2 \frac{\partial u}{\partial z} \frac{\partial^{2} u}{\partial z \partial t}+2 \frac{\partial v}{\partial z} \frac{\partial^{2} v}{\partial z \partial t}\right\}+\frac{\gamma_{1}}{\rho} \frac{\partial}{\partial z}\left\{+2 \frac{\partial}{\partial t}\left(\frac{\partial u}{\partial z} \frac{\partial^{2} u}{\partial z \partial t}+\frac{\partial v}{\partial z} \frac{\partial^{2} v}{\partial z \partial t}\right)\right\} \\
& \left.+2\left(\frac{\partial u}{\partial z} \frac{\partial^{3} u}{\partial z \partial t^{2}}+\frac{\partial v}{\partial z} \frac{\partial^{3} v}{\partial z \partial t^{2}}\right)\right) \\
& +\frac{\gamma_{3}}{\rho} \frac{\partial}{\partial z}\left\{\begin{array}{l}
\left(\frac{\partial^{2} u}{\partial z \partial t}\right)^{2}+\left(\frac{\partial^{2} v}{\partial z \partial t}\right)^{2} \\
+4\left(\left(\frac{\partial u}{\partial z}\right)^{2}+\left(\frac{\partial v}{\partial z}\right)^{2}\right)^{2}
\end{array}\right\}+\frac{\left(4 \gamma_{4}+4 \gamma_{5}+2 \gamma_{6}\right.}{\rho} \frac{\partial}{\partial z}\left(\left(\frac{\partial u}{\partial z}\right)^{2}+\left(\frac{\partial v}{\partial z}\right)^{2}\right)^{2} .
\end{aligned}
$$

Defining the modified pressure 


$$
\begin{aligned}
& \hat{p}=\frac{p}{\rho}-\frac{\left(2 \alpha_{1}+\alpha_{2}\right)}{\rho}\left\{\left(\frac{\partial u}{\partial z}\right)^{2}+\left(\frac{\partial v}{\partial z}\right)^{2}\right\}-\frac{\beta_{1}}{\rho}\left\{\begin{array}{l}
2 \frac{\partial}{\partial t}\left(\left(\frac{\partial u}{\partial z}\right)^{2}+\left(\frac{\partial v}{\partial z}\right)^{2}\right) \\
+2 \frac{\partial u}{\partial z} \frac{\partial^{2} u}{\partial z \partial t}+2 \frac{\partial v}{\partial z} \frac{\partial^{2} v}{\partial z \partial t}
\end{array}\right\} \\
& -\frac{\beta_{2}}{\rho}\left\{2 \frac{\partial u}{\partial z} \frac{\partial^{2} u}{\partial z \partial t}+2 \frac{\partial v}{\partial z} \frac{\partial^{2} v}{\partial z \partial t}\right\}-\frac{\gamma_{1}}{\rho}\left\{\begin{array}{c}
2 \frac{\partial^{2}}{\partial t^{2}}\left(\left(\frac{\partial u}{\partial z}\right)^{2}+\left(\frac{\partial v}{\partial z}\right)^{2}\right) \\
+2 \frac{\partial}{\partial t}\left(\frac{\partial u}{\partial z} \frac{\partial^{2} u}{\partial z \partial t}+\frac{\partial v}{\partial z} \frac{\partial^{2} v}{\partial z \partial t}\right) \\
+2\left(\frac{\partial u}{\partial z} \frac{\partial^{3} u}{\partial z \partial t^{2}}+\frac{\partial v}{\partial z} \frac{\partial^{3} v}{\partial z \partial t^{2}}\right)
\end{array}\right\} \\
& -\frac{\gamma_{3}}{\rho}\left\{\begin{array}{l}
\left(\frac{\partial^{2} u}{\partial z \partial t}\right)^{2}+\left(\frac{\partial^{2} v}{\partial z \partial t}\right)^{2} \\
+4\left(\left(\frac{\partial u}{\partial z}\right)^{2}+\left(\frac{\partial v}{\partial z}\right)^{2}\right)^{2}
\end{array}\right\}-\frac{\left(4 \gamma_{4}+4 \gamma_{5}+2 \gamma_{6}\right)}{\rho}\left(\left(\frac{\partial u}{\partial z}\right)^{2}+\left(\frac{\partial v}{\partial z}\right)^{2}\right)^{2},
\end{aligned}
$$

then Eqs. (15)-(17) become

$$
\begin{aligned}
\frac{\partial u}{\partial t}-2 \Omega v-\Omega^{2} x & =-\frac{\partial \hat{p}}{\partial x}+\frac{\mu}{\rho} \frac{\partial^{2} u}{\partial z^{2}}+\frac{\alpha_{1}}{\rho} \frac{\partial^{3} u}{\partial z^{2} \partial t}+\frac{\beta_{1}}{\rho} \frac{\partial^{4} u}{\partial z^{2} \partial t^{2}} \\
& +\frac{2\left(\beta_{2}+\beta_{3}\right)}{\rho} \frac{\partial}{\partial z}\left[2 \frac{\partial u}{\partial z}\left\{\left(\frac{\partial u}{\partial z}\right)^{2}+\left(\frac{\partial v}{\partial z}\right)^{2}\right\}\right] \\
& +\frac{\gamma_{2}}{\rho} \frac{\partial}{\partial z}\left[\frac{\partial u}{\partial z}\left\{2 \frac{\partial}{\partial t}\left(\left(\frac{\partial u}{\partial z}\right)^{2}+\left(\frac{\partial v}{\partial z}\right)^{2}\right)+2 \frac{\partial u}{\partial z} \frac{\partial^{2} u}{\partial z \partial t}+2 \frac{\partial v}{\partial z} \frac{\partial^{2} v}{\partial z \partial t}\right\}\right] \\
& +\frac{\left(\gamma_{3}+\gamma_{5}\right)}{\rho} \frac{\partial}{\partial z}\left[2 \frac{\partial^{2} u}{\partial z \partial t}\left\{\left(\frac{\partial u}{\partial z}\right)^{2}+\left(\frac{\partial v}{\partial z}\right)^{2}\right\}\right] \\
& +\frac{\partial}{\partial z}\left[\frac{\partial u}{\partial z}\left\{2 \frac{\gamma_{7}}{\rho} \frac{\partial}{\partial t}\left(\left(\frac{\partial u}{\partial z}\right)^{2}+\left(\frac{\partial v}{\partial z}\right)^{2}\right)+\frac{\left(\gamma_{7}+\gamma_{8}\right)}{\rho}\left(\frac{\partial u}{\partial z} \frac{\partial^{2} u}{\partial z \partial t}+\frac{\partial v}{\partial z} \frac{\partial^{2} v}{\partial z \partial t}\right)\right\}\right] \\
& +\frac{\gamma_{1}}{\rho} \frac{\partial^{5} u}{\partial z^{2} \partial t^{3}}-\frac{\sigma}{\rho} B_{0}^{2} u,
\end{aligned}
$$




$$
\begin{aligned}
\frac{\partial v}{\partial t}+2 \Omega u-\Omega^{2} y & =-\frac{\partial \hat{p}}{\partial y}+\frac{\mu}{\rho} \frac{\partial^{2} v}{\partial z^{2}}+\frac{\alpha_{1}}{\rho} \frac{\partial^{3} v}{\partial z^{2} \partial t}+\frac{\beta_{1}}{\rho} \frac{\partial^{4} v}{\partial z^{2} \partial t^{2}} \\
& +\frac{2\left(\beta_{2}+\beta_{3}\right)}{\rho} \frac{\partial}{\partial z}\left[2 \frac{\partial v}{\partial z}\left\{\left(\frac{\partial u}{\partial z}\right)^{2}+\left(\frac{\partial v}{\partial z}\right)^{2}\right\}\right] \\
& +\frac{\gamma_{2}}{\rho} \frac{\partial}{\partial z}\left[\frac{\partial v}{\partial z}\left\{2 \frac{\partial}{\partial t}\left(\left(\frac{\partial u}{\partial z}\right)^{2}+\left(\frac{\partial v}{\partial z}\right)^{2}\right)+2 \frac{\partial u}{\partial z} \frac{\partial^{2} u}{\partial z \partial t}+2 \frac{\partial v}{\partial z} \frac{\partial^{2} v}{\partial z \partial t}\right\}\right] \\
& +\frac{\left(\gamma_{3}+\gamma_{5}\right)}{\rho} \frac{\partial}{\partial z}\left[2 \frac{\partial^{2} v}{\partial z \partial t}\left\{\left(\frac{\partial u}{\partial z}\right)^{2}+\left(\frac{\partial v}{\partial z}\right)^{2}\right\}\right] \\
& +\frac{\partial}{\partial z}\left[\frac{\partial v}{\partial z}\left\{2 \frac{\gamma_{7}}{\rho} \frac{\partial}{\partial t}\left(\left(\frac{\partial u}{\partial z}\right)^{2}+\left(\frac{\partial v}{\partial z}\right)^{2}\right)+\frac{\left(\gamma_{7}+\gamma_{8}\right)}{\rho}\left(\frac{\partial u}{\partial z} \frac{\partial^{2} u}{\partial z \partial t}+\frac{\partial v}{\partial z} \frac{\partial^{2} v}{\partial z \partial t}\right)\right\}\right] \\
& +\frac{\gamma_{1}}{\rho} \frac{\partial^{5} v}{\partial z^{2} \partial t^{3}}-\frac{\sigma}{\rho} B_{0}^{2} v
\end{aligned}
$$

Since $r^{2}=x^{2}+y^{2}$, therefore $x=\frac{\partial}{\partial x}\left(\frac{1}{2} r^{2}\right)$ and $y=\frac{\partial}{\partial y}\left(\frac{1}{2} r^{2}\right)$. In view these substitutions we can write Eqs. (19)-(21) in the following manner:

$$
\begin{aligned}
\frac{\partial u}{\partial t}-2 \Omega v= & -\frac{\partial}{\partial x}\left(\hat{p}-\frac{1}{2} \Omega^{2} r^{2}\right)+\frac{\mu}{\rho} \frac{\partial^{2} u}{\partial z^{2}}+\frac{\alpha_{1}}{\rho} \frac{\partial^{3} u}{\partial z^{2} \partial t}+\frac{\beta_{1}}{\rho} \frac{\partial^{4} u}{\partial z^{2} \partial t^{2}} \\
& +\frac{2\left(\beta_{2}+\beta_{3}\right)}{\rho} \frac{\partial}{\partial z}\left[2 \frac{\partial u}{\partial z}\left\{\left(\frac{\partial u}{\partial z}\right)^{2}+\left(\frac{\partial v}{\partial z}\right)^{2}\right\}\right] \\
& +\frac{\gamma_{2}}{\rho} \frac{\partial}{\partial z}\left[\frac{\partial u}{\partial z}\left\{2 \frac{\partial}{\partial t}\left(\left(\frac{\partial u}{\partial z}\right)^{2}+\left(\frac{\partial v}{\partial z}\right)^{2}\right)+2 \frac{\partial u}{\partial z} \frac{\partial^{2} u}{\partial z \partial t}+2 \frac{\partial v}{\partial z} \frac{\partial^{2} v}{\partial z \partial t}\right\}\right] \\
& +\frac{\left(\gamma_{3}+\gamma_{5}\right)}{\rho} \frac{\partial}{\partial z}\left[2 \frac{\partial^{2} u}{\partial z \partial t}\left\{\left(\frac{\partial u}{\partial z}\right)^{2}+\left(\frac{\partial v}{\partial z}\right)^{2}\right\}\right] \\
& +\frac{\partial}{\partial z}\left[\frac{\partial u}{\partial z}\left\{2 \frac{\gamma_{7}}{\rho} \frac{\partial}{\partial t}\left(\left(\frac{\partial u}{\partial z}\right)^{2}+\left(\frac{\partial v}{\partial z}\right)^{2}\right)+\frac{\left(\gamma_{7}+\gamma_{8}\right)}{\rho}\left(\frac{\partial u}{\partial z} \frac{\partial^{2} u}{\partial z \partial t}+\frac{\partial v}{\partial z} \frac{\partial^{2} v}{\partial z \partial t}\right)\right\}\right] \\
& +\frac{\gamma_{1}}{\rho} \frac{\partial^{5} u}{\partial z^{2} \partial t^{3}}-\frac{\sigma}{\rho} B_{0}^{2} u
\end{aligned}
$$




$$
\begin{aligned}
\frac{\partial v}{\partial t}+2 \Omega u= & -\frac{\partial}{\partial y}\left(\hat{p}-\frac{1}{2} \Omega^{2} r^{2}\right)+\frac{\mu}{\rho} \frac{\partial^{2} v}{\partial z^{2}}+\frac{\alpha_{1}}{\rho} \frac{\partial^{3} v}{\partial z^{2} \partial t}+\frac{\beta_{1}}{\rho} \frac{\partial^{4} v}{\partial z^{2} \partial t^{2}} \\
& +\frac{2\left(\beta_{2}+\beta_{3}\right)}{\rho} \frac{\partial}{\partial z}\left[2 \frac{\partial v}{\partial z}\left\{\left(\frac{\partial u}{\partial z}\right)^{2}+\left(\frac{\partial v}{\partial z}\right)^{2}\right\}\right] \\
& +\frac{\gamma_{2}}{\rho} \frac{\partial}{\partial z}\left[\frac{\partial v}{\partial z}\left\{2 \frac{\partial}{\partial t}\left(\left(\frac{\partial u}{\partial z}\right)^{2}+\left(\frac{\partial v}{\partial z}\right)^{2}\right)+2 \frac{\partial u}{\partial z} \frac{\partial^{2} u}{\partial z \partial t}+2 \frac{\partial v}{\partial z} \frac{\partial^{2} v}{\partial z \partial t}\right\}\right] \\
& +\frac{\left(\gamma_{3}+\gamma_{5}\right)}{\rho} \frac{\partial}{\partial z}\left[2 \frac{\partial^{2} v}{\partial z \partial t}\left\{\left(\frac{\partial u}{\partial z}\right)^{2}+\left(\frac{\partial v}{\partial z}\right)^{2}\right\}\right] \\
& +\frac{\partial}{\partial z}\left[\frac{\partial v}{\partial z}\left\{2 \frac{\gamma_{7}}{\rho} \frac{\partial}{\partial t}\left(\left(\frac{\partial u}{\partial z}\right)^{2}+\left(\frac{\partial v}{\partial z}\right)^{2}\right)+\frac{\left(\gamma_{7}+\gamma_{8}\right)}{\rho}\left(\frac{\partial u}{\partial z} \frac{\partial^{2} u}{\partial z \partial t}+\frac{\partial v}{\partial z} \frac{\partial^{2} v}{\partial z \partial t}\right)\right\}\right] \\
& +\frac{\gamma_{1}}{\rho} \frac{\partial^{5} v}{\partial z^{2} \partial t^{3}}-\frac{\sigma}{\rho} B_{0}^{2} v, \\
0 & =-\frac{\partial}{\partial z}\left(\hat{p}-\frac{1}{2} \Omega^{2} r^{2}\right) .
\end{aligned}
$$

Redefining the modified pressure

$$
\tilde{p}=\hat{p}-\frac{1}{2} \Omega^{2} r^{2},
$$

Eqs. (22)-(24) become

$$
\begin{aligned}
\frac{\partial u}{\partial t}-2 \Omega v=-\frac{\partial \tilde{p}}{\partial x} & +\frac{\mu}{\rho} \frac{\partial^{2} u}{\partial z^{2}}+\frac{\alpha_{1}}{\rho} \frac{\partial^{3} u}{\partial z^{2} \partial t}+\frac{\beta_{1}}{\rho} \frac{\partial^{4} u}{\partial z^{2} \partial t^{2}} \\
& +\frac{2\left(\beta_{2}+\beta_{3}\right)}{\rho} \frac{\partial}{\partial z}\left[2 \frac{\partial u}{\partial z}\left\{\left(\frac{\partial u}{\partial z}\right)^{2}+\left(\frac{\partial v}{\partial z}\right)^{2}\right\}\right] \\
& +\frac{\gamma_{2}}{\rho} \frac{\partial}{\partial z}\left[\frac{\partial u}{\partial z}\left\{2 \frac{\partial}{\partial t}\left(\left(\frac{\partial u}{\partial z}\right)^{2}+\left(\frac{\partial v}{\partial z}\right)^{2}\right)+2 \frac{\partial u}{\partial z} \frac{\partial^{2} u}{\partial z \partial t}+2 \frac{\partial v}{\partial z} \frac{\partial^{2} v}{\partial z \partial t}\right\}\right] \\
& +\frac{\left(\gamma_{3}+\gamma_{5}\right)}{\rho} \frac{\partial}{\partial z}\left[2 \frac{\partial^{2} u}{\partial z \partial t}\left\{\left(\frac{\partial u}{\partial z}\right)^{2}+\left(\frac{\partial v}{\partial z}\right)^{2}\right\}\right] \\
& +\frac{\partial}{\partial z}\left[\frac{\partial u}{\partial z}\left\{2 \frac{\gamma_{7}}{\rho} \frac{\partial}{\partial t}\left(\left(\frac{\partial u}{\partial z}\right)^{2}+\left(\frac{\partial v}{\partial z}\right)^{2}\right)+\frac{\left(\gamma_{7}+\gamma_{8}\right)}{\rho}\left(\frac{\partial u}{\partial z} \frac{\partial^{2} u}{\partial z \partial t}+\frac{\partial v}{\partial z} \frac{\partial^{2} v}{\partial z \partial t}\right)\right\}\right] \\
& +\frac{\gamma_{1}}{\rho} \frac{\partial^{5} u}{\partial z^{2} \partial t^{3}}-\frac{\sigma}{\rho} B_{0}^{2} u,
\end{aligned}
$$




$$
\begin{aligned}
\frac{\partial v}{\partial t}+2 \Omega u=-\frac{\partial \tilde{p}}{\partial y} & +\frac{\mu}{\rho} \frac{\partial^{2} v}{\partial z^{2}}+\frac{\alpha_{1}}{\rho} \frac{\partial^{3} v}{\partial z^{2} \partial t}+\frac{\beta_{1}}{\rho} \frac{\partial^{4} v}{\partial z^{2} \partial t^{2}} \\
& +\frac{2\left(\beta_{2}+\beta_{3}\right)}{\rho} \frac{\partial}{\partial z}\left[2 \frac{\partial v}{\partial z}\left\{\left(\frac{\partial u}{\partial z}\right)^{2}+\left(\frac{\partial v}{\partial z}\right)^{2}\right\}\right] \\
& +\frac{\gamma_{2}}{\rho} \frac{\partial}{\partial z}\left[\frac{\partial v}{\partial z}\left\{2 \frac{\partial}{\partial t}\left(\left(\frac{\partial u}{\partial z}\right)^{2}+\left(\frac{\partial v}{\partial z}\right)^{2}\right)+2 \frac{\partial u}{\partial z} \frac{\partial^{2} u}{\partial z \partial t}+2 \frac{\partial v}{\partial z} \frac{\partial^{2} v}{\partial z \partial t}\right\}\right] \\
& \left.+\frac{\left(\gamma_{3}+\gamma_{5}\right)}{\rho} \frac{\partial}{\partial z}\left[2 \frac{\partial^{2} v}{\partial z \partial t}\left\{\left(\frac{\partial u}{\partial z}\right)^{2}+\left(\frac{\partial v}{\partial z}\right)^{2}\right\}\right]\right] \\
& +\frac{\partial}{\partial z}\left[\frac{\partial v}{\partial z}\left\{2 \frac{\gamma_{7}}{\rho} \frac{\partial}{\partial t}\left(\left(\frac{\partial u}{\partial z}\right)^{2}+\left(\frac{\partial v}{\partial z}\right)^{2}\right)+\frac{\left(\gamma_{7}+\gamma_{8}\right)}{\rho}\left(\frac{\partial u}{\partial z} \frac{\partial^{2} u}{\partial z \partial t}+\frac{\partial v}{\partial z} \frac{\partial^{2} v}{\partial z \partial t}\right)\right\}\right] \\
& +\frac{\gamma_{1}}{\rho} \frac{\partial^{5} v}{\partial z^{2} \partial t^{3}}-\frac{\sigma}{\rho} B_{0}^{2} v
\end{aligned}
$$

Differentiating Eqs. (26) and (27) with respect to $\mathrm{z}$ and making use of Eq. (28), and then integrating with respect to $\mathrm{z}$ to obtain

$$
\begin{aligned}
\frac{\partial u}{\partial t}-2 \Omega v=\frac{\mu}{\rho} & \frac{\partial^{2} u}{\partial z^{2}}+\frac{\alpha_{1}}{\rho} \frac{\partial^{3} u}{\partial z^{2} \partial t}+\frac{\beta_{1}}{\rho} \frac{\partial^{4} u}{\partial z^{2} \partial t^{2}} \\
& +\frac{2\left(\beta_{2}+\beta_{3}\right)}{\rho} \frac{\partial}{\partial z}\left[2 \frac{\partial u}{\partial z}\left\{\left(\frac{\partial u}{\partial z}\right)^{2}+\left(\frac{\partial v}{\partial z}\right)^{2}\right\}\right] \\
& +\frac{\gamma_{2}}{\rho} \frac{\partial}{\partial z}\left[\frac{\partial u}{\partial z}\left\{2 \frac{\partial}{\partial t}\left(\left(\frac{\partial u}{\partial z}\right)^{2}+\left(\frac{\partial v}{\partial z}\right)^{2}\right)+2 \frac{\partial u}{\partial z} \frac{\partial^{2} u}{\partial z \partial t}+2 \frac{\partial v}{\partial z} \frac{\partial^{2} v}{\partial z \partial t}\right\}\right] \\
& +\frac{\left(\gamma_{3}+\gamma_{5}\right)}{\rho} \frac{\partial}{\partial z}\left[2 \frac{\partial^{2} u}{\partial z \partial t}\left\{\left(\frac{\partial u}{\partial z}\right)^{2}+\left(\frac{\partial v}{\partial z}\right)^{2}\right\}\right] \\
& +\frac{\partial}{\partial z}\left[\frac{\partial u}{\partial z}\left\{2 \frac{\gamma_{7}}{\rho} \frac{\partial}{\partial t}\left(\left(\frac{\partial u}{\partial z}\right)^{2}+\left(\frac{\partial v}{\partial z}\right)^{2}\right)+\frac{\left(\gamma_{7}+\gamma_{8}\right)}{\rho}\left(\frac{\partial u}{\partial z} \frac{\partial^{2} u}{\partial z \partial t}+\frac{\partial v}{\partial z} \frac{\partial^{2} v}{\partial z \partial t}\right)\right\}\right] \\
& +\frac{\gamma_{1}}{\rho} \frac{\partial^{5} u}{\partial z^{2} \partial t^{3}}-\frac{\sigma}{\rho} B_{0}^{2} u+\lambda(t),
\end{aligned}
$$




$$
\begin{aligned}
\frac{\partial v}{\partial t}+2 \Omega u=\frac{\mu}{\rho} \frac{\partial^{2} v}{\partial z^{2}} & +\frac{\alpha_{1}}{\rho} \frac{\partial^{3} v}{\partial z^{2} \partial t}+\frac{\beta_{1}}{\rho} \frac{\partial^{4} v}{\partial z^{2} \partial t^{2}} \\
& +\frac{2\left(\beta_{2}+\beta_{3}\right)}{\rho} \frac{\partial}{\partial z}\left[2 \frac{\partial v}{\partial z}\left\{\left(\frac{\partial u}{\partial z}\right)^{2}+\left(\frac{\partial v}{\partial z}\right)^{2}\right\}\right] \\
& +\frac{\gamma_{2}}{\rho} \frac{\partial}{\partial z}\left[\frac{\partial v}{\partial z}\left\{2 \frac{\partial}{\partial t}\left(\left(\frac{\partial u}{\partial z}\right)^{2}+\left(\frac{\partial v}{\partial z}\right)^{2}\right)+2 \frac{\partial u}{\partial z} \frac{\partial^{2} u}{\partial z \partial t}+2 \frac{\partial v}{\partial z} \frac{\partial^{2} v}{\partial z \partial t}\right\}\right] \\
& \left.+\frac{\left(\gamma_{3}+\gamma_{5}\right)}{\rho} \frac{\partial}{\partial z}\left[2 \frac{\partial^{2} v}{\partial z \partial t}\left\{\left(\frac{\partial u}{\partial z}\right)^{2}+\left(\frac{\partial v}{\partial z}\right)^{2}\right\}\right]\right] \\
& +\frac{\partial}{\partial z}\left[\frac{\partial v}{\partial z}\left\{2 \frac{\gamma_{7}}{\rho} \frac{\partial}{\partial t}\left(\left(\frac{\partial u}{\partial z}\right)^{2}+\left(\frac{\partial v}{\partial z}\right)^{2}\right)+\frac{\left(\gamma_{7}+\gamma_{8}\right)}{\rho}\left(\frac{\partial u}{\partial z} \frac{\partial^{2} u}{\partial z \partial t}+\frac{\partial v}{\partial z} \frac{\partial^{2} v}{\partial z \partial t}\right)\right\}\right] \\
& +\frac{\gamma_{1}}{\rho} \frac{\partial^{5} v}{\partial z^{2} \partial t^{3}}-\frac{\sigma}{\rho} B_{0}^{2} v+\delta(t) .
\end{aligned}
$$

On multiplying Eq. (30) by $i$ and then adding the resulting equation in Eq. (29) we get

$$
\begin{aligned}
\frac{\partial q}{\partial t}+2 i \Omega q=\frac{\mu}{\rho} & \frac{\partial^{2} q}{\partial z^{2}}+\frac{\alpha_{1}}{\rho} \frac{\partial^{3} q}{\partial z^{2} \partial t}+\frac{\beta_{1}}{\rho} \frac{\partial^{4} q}{\partial z^{2} \partial t^{2}} \\
& +\frac{4\left(\beta_{2}+\beta_{3}\right)}{\rho} \frac{\partial}{\partial z}\left[\left\{\left(\frac{\partial q}{\partial z}\right)^{2}\left(\frac{\partial \bar{q}}{\partial z}\right)\right\}\right] \\
& +\frac{\gamma_{2}}{\rho} \frac{\partial}{\partial z}\left[\left\{3\left(\frac{\partial q}{\partial z}\right) \frac{\partial}{\partial t}\left(\frac{\partial q}{\partial z} \frac{\partial \bar{q}}{\partial z}\right)\right\}\right]+\frac{\gamma_{1}}{\rho} \frac{\partial^{5} q}{\partial z^{3} \partial t^{2}} \\
& +\frac{\left(\gamma_{3}+\gamma_{5}\right)}{\rho} \frac{\partial}{\partial z}\left[\left\{2\left(\frac{\partial^{2} q}{\partial z \partial t}\right)\left(\frac{\partial q}{\partial z} \frac{\partial \bar{q}}{\partial z}\right)\right\}\right] \\
& +\frac{\partial}{\partial z}\left[\frac{2 \gamma_{7}}{\rho}\left(\frac{\partial q}{\partial z}\right) \frac{\partial}{\partial t}\left(\frac{\partial q}{\partial z} \frac{\partial \bar{q}}{\partial z}\right)\right. \\
\left.+\frac{\left(\gamma_{7}+\gamma_{8}\right.}{2}\right) & {\left.\left[\frac{\partial q}{\partial z}\right) \frac{\partial}{\partial t}\left(\frac{\partial q}{\partial z} \frac{\partial \bar{q}}{\partial z}\right)\right]-\frac{\sigma}{\rho} B_{0}^{2} q+\psi(t), }
\end{aligned}
$$

where 


$$
q=u+i v, \quad \bar{q}=u-i v, \quad \psi(t)=\lambda(t)+i \delta(t)
$$

For steady state the Equations (29) and (30) reduce to

$$
\begin{aligned}
& -2 \Omega v=\frac{\mu}{\rho} \frac{\partial^{2} u}{\partial z^{2}}+\frac{2\left(\beta_{2}+\beta_{3}\right)}{\rho} \frac{\partial}{\partial z}\left[2 \frac{\partial u}{\partial z}\left\{\left(\frac{\partial u}{\partial z}\right)^{2}+\left(\frac{\partial v}{\partial z}\right)^{2}\right\}\right]-\frac{\sigma}{\rho} B_{0}^{2} u, \\
& 2 \Omega u=\frac{\mu}{\rho} \frac{\partial^{2} v}{\partial z^{2}}+\frac{2\left(\beta_{2}+\beta_{3}\right)}{\rho} \frac{\partial}{\partial z}\left[2 \frac{\partial v}{\partial z}\left\{\left(\frac{\partial u}{\partial z}\right)^{2}+\left(\frac{\partial v}{\partial z}\right)^{2}\right\}\right]-\frac{\sigma}{\rho} B_{0}^{2} v,
\end{aligned}
$$

Introducing the dimensionless variables

$$
z^{*}=\frac{z}{L}, u^{*}=\frac{u L}{v}, \text { and } v^{*}=\frac{v L}{v}
$$

in above equations and simplifying the resulting equations and dropping ${ }^{\text {*' }}$ to obtain

$$
\begin{aligned}
& -2 v E^{-1}=\frac{\partial^{2} u}{\partial z^{2}}+4 \beta\left[3\left(\frac{\partial^{2} u}{\partial z^{2}}\right)\left(\frac{\partial u}{\partial z}\right)^{2}+\left(\frac{\partial^{2} u}{\partial z^{2}}\right)\left(\frac{\partial v}{\partial z}\right)^{2}+2\left(\frac{\partial u}{\partial z}\right)\left(\frac{\partial v}{\partial z}\right)\left(\frac{\partial^{2} v}{\partial z^{2}}\right)\right]-H^{2} u \\
& 2 E^{-1} u=\frac{\partial^{2} v}{\partial z^{2}}+4 \beta\left[3\left(\frac{\partial^{2} v}{\partial z^{2}}\right)\left(\frac{\partial v}{\partial z}\right)^{2}+\left(\frac{\partial^{2} v}{\partial z^{2}}\right)\left(\frac{\partial u}{\partial z}\right)^{2}+2\left(\frac{\partial u}{\partial z}\right)\left(\frac{\partial v}{\partial z}\right)\left(\frac{\partial^{2} u}{\partial z^{2}}\right)\right]-H^{2} v
\end{aligned}
$$

where

$$
E^{-1}=\frac{\Omega L^{2}}{v}, \beta=\frac{4\left(\beta_{2}+\beta_{3}\right) v}{\rho L^{4}}, H^{2}=\frac{n L^{2}}{v}, n=\frac{\sigma B_{0}^{2}}{\rho}
$$

and $E$ is the Ekman number while $\mathrm{H}$ is the Hartmann number.

\section{Numerical procedure}

Consider a simplest boundary value problem

$$
\begin{gathered}
F\left(u^{\prime \prime}, u^{\prime}, u, z\right)=0, \\
u(a)=A \text { and } u(b)=B .
\end{gathered}
$$

To solve the boundary value problem the derivatives $u^{\prime}$ and $u^{\prime \prime}$ involved in the problem are approximated by finite differences of appropriate order. If we employ second order central difference formulation, then we can write

$$
u^{\prime}(z)=\frac{u(z+h)-u(z-h)}{2 h}+\mathrm{O}\left(h^{2}\right)
$$




$$
u^{\prime \prime}(z)=\frac{u(z+h)-2 u(z)+u(z-h)}{h^{2}}+\mathrm{O}\left(h^{2}\right)
$$

This converts the given boundary value problem into a linear system of equations involving values of the function $u$ at $a, a+h, a+2 h, \cdots, \mathrm{b}$. For higher accuracy, one should choose $h$ small. However, this increases the number of equations in the system which in turn increases the computational time.

Depending upon the size of this resulting system of linear equations, it can either be solved by exact methods or approximate methods.

In the present problem the governing differential equations (36) and (37) are highly nonlinear which cannot be solved analytically. These equations are discretized using second order central finite difference approximations defined in Eqs. (41) and (42). The resulting system of algebraic equations is solved using successive under relaxation scheme. The difference equations are linearized employing a procedure known as lagging the coefficients [59]. The iterative procedure is repeated until convergence is obtained according to the following criterion

$$
\max \left|u^{(n+1)}-u^{(n)}\right|<\varepsilon,
$$

where superscript ' $n$ ' represents the number of iteration and ' $\varepsilon$ ' is the order of accuracy. In the present case $\varepsilon$ is taken as $10^{-8}$.

\section{Numerical results and discussion}

The steady velocity components $u$ and $v$ are plotted against independent variable $z$ for different values of Ekman number $E$, Hartmann number $H$ and material parameter $\beta$ and results are compared for two types of fluids: the Newtonian fluid, for which $\beta_{i}=0(i=1,2,3)$, and the non-Newtonian fluid, in which we choose $\beta=1$. Fig. 1 shows the effect of Hartmann number $H$ on the velocity components $u$ and $v$. We fixed $E=1$ and varied $H=0,1,3,5$. It is observed that an increase in the Hartmann number reduces the velocity components $u$ and $v$ due to the effects of the magnetic force against the flow direction. Figs. 1a and $1 b$ show that with an increase of Hartmann number $H$, the curvature of the velocity component $u$ profile increases for both a Newtonian fluid and non-Newtonian fluid. Quite contrary, increasing Hartmann number $H$ causes the velocity component $v$ profile to become less parabolic, see Figs. 1c and 1d. It is also noted that decrease in $u$ and $v$ in the Newtonian fluid is larger as compared with non-Newtonian fluid. Furthermore, the boundary layer thickness is drastically decreased by increasing $H$. It means that the magnetic field provides some mechanism to control the boundary layer thickness.

The dependence of the velocity components $u$ and $v$ on the Ekman number is shown in Fig. 2. In Fig. 2 we fixed $H=1$ and varied $E=0.1,0.2,0.3$. It is observed that velocity component $u$ increases with an increase in Ekman number $E$ for the Newtonian fluid while it remains almost unaffected for non-Newtonian fluid (Figs. 2a, 2b). Moreover, a 


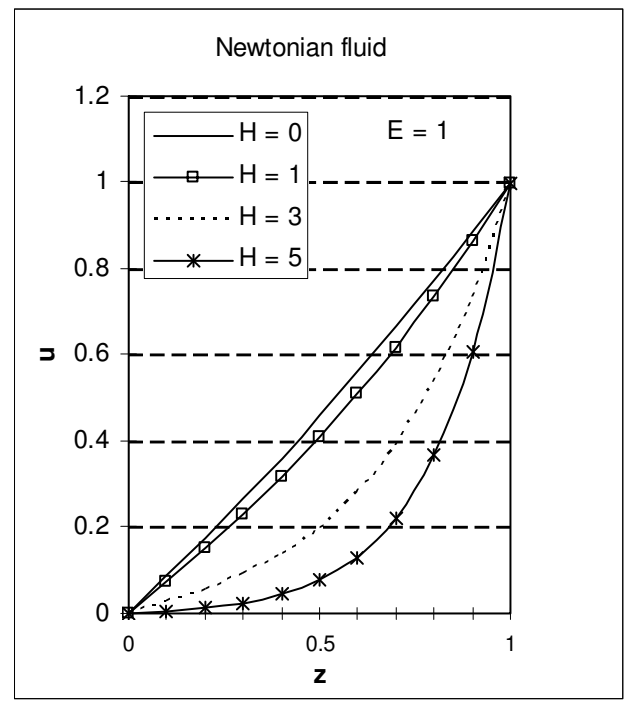

(a)

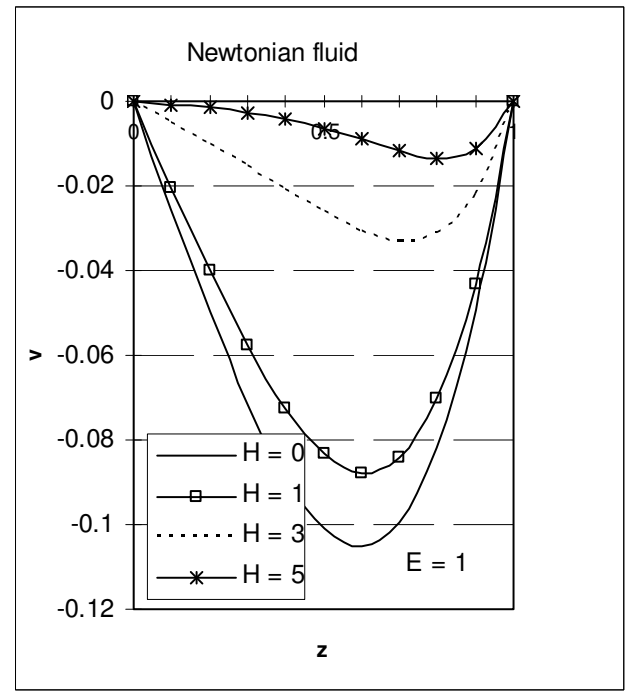

(c)

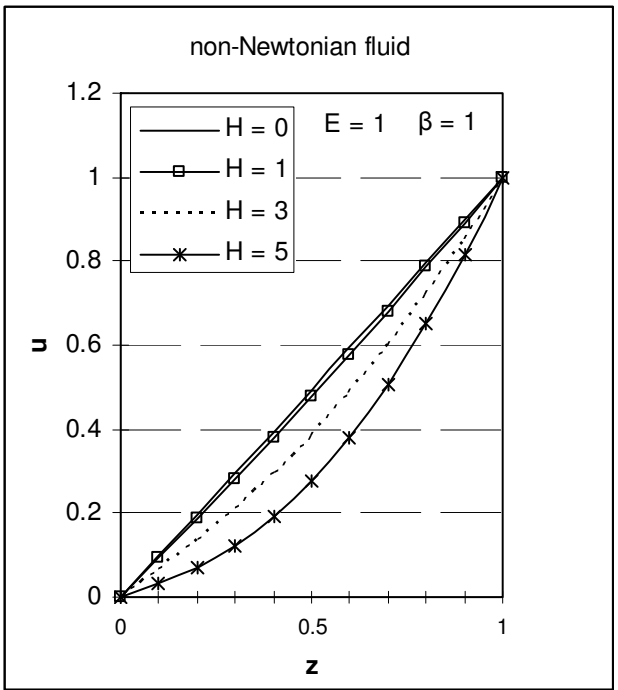

(b)

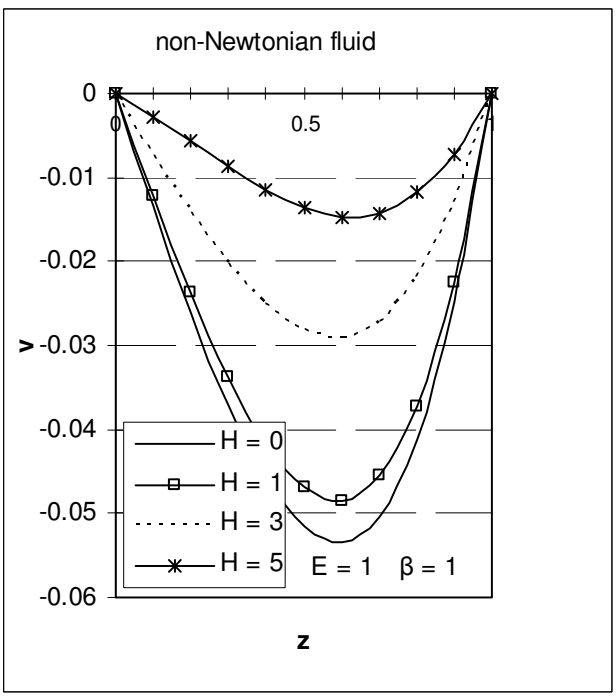

(d)

Fig. 1. Variation of velocity components $u$ and $v$ with $\mathrm{z}$ for $H=0,1,3,5 ; E=1, \beta=0$ in (a), (c); $E=1, \beta=1$ in (b), (d).

backflow is observed near the boundary $z=0$ for $E=0.1$. On the contrary, the magnitude of velocity component $v$ decreases with an increase in Ekman number $E$ for the both types of the fluids (Figs. 2c, 2d). This velocity component has larger magnitude in Newtonian fluid as compared with non-Newtonian fluid. 


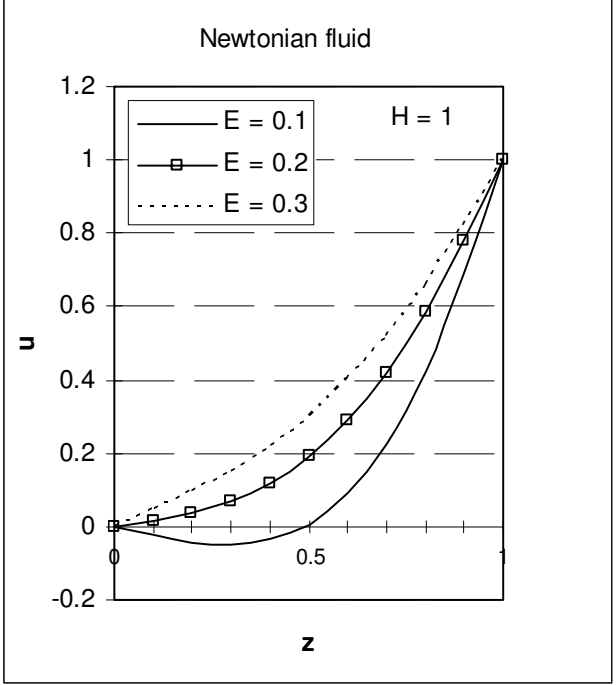

(a)

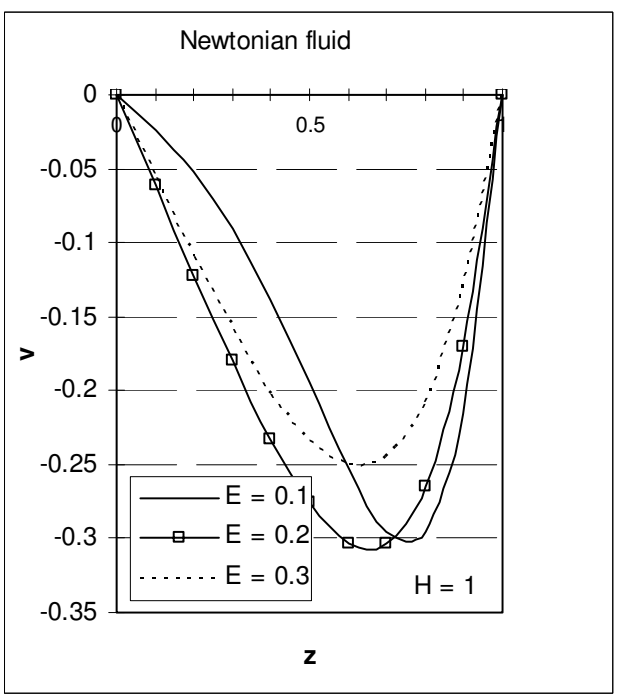

(c)

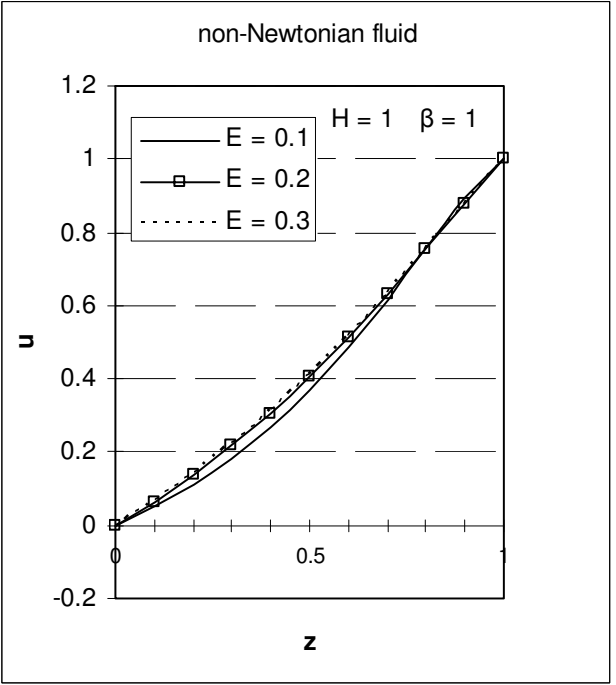

(b)

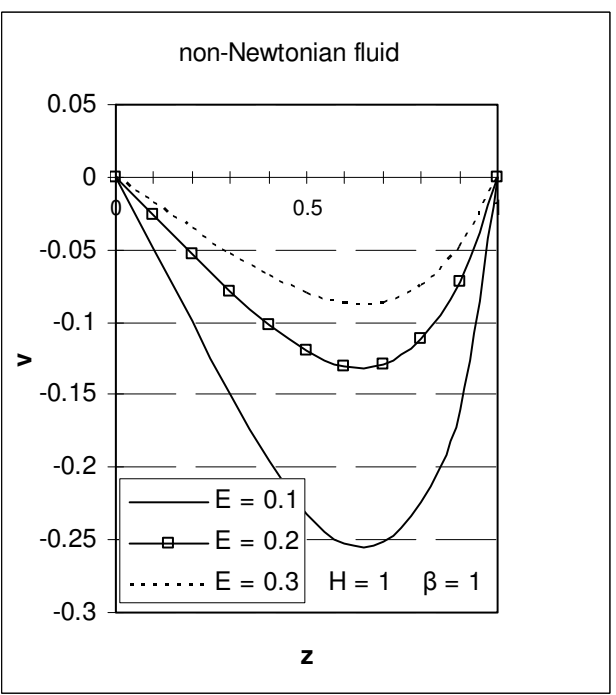

(d)

Fig. 2. Variation of velocity components $u$ and $v$ with $z$ for $E=0.1,0.2,0.3 ; H=1, \beta=0$ in (a), (c); $H=1, \beta=1$ in (b), (d).

Figs. 3,4 depict the variation of the velocity components $u$ and $v$ with $z$ for various values of material parameter $\beta$ fixing $E=1$ and taking $H=1$ in Figs. $3 a$ and $3 c$, while $H=5$ in $3 b$, $3 \mathrm{~d}$, and in Fig. 4. It is observed from Fig. $3 \mathrm{~b}$ and $4 \mathrm{a}$ that when the material parameter $\beta$ increases from $\beta=1$ to a large value of 20 , the velocity component $u$ tend to approach the 


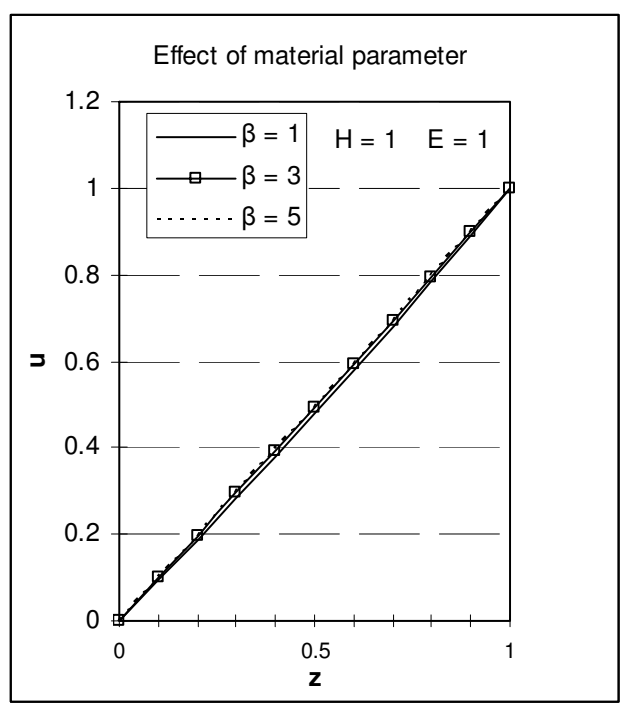

a

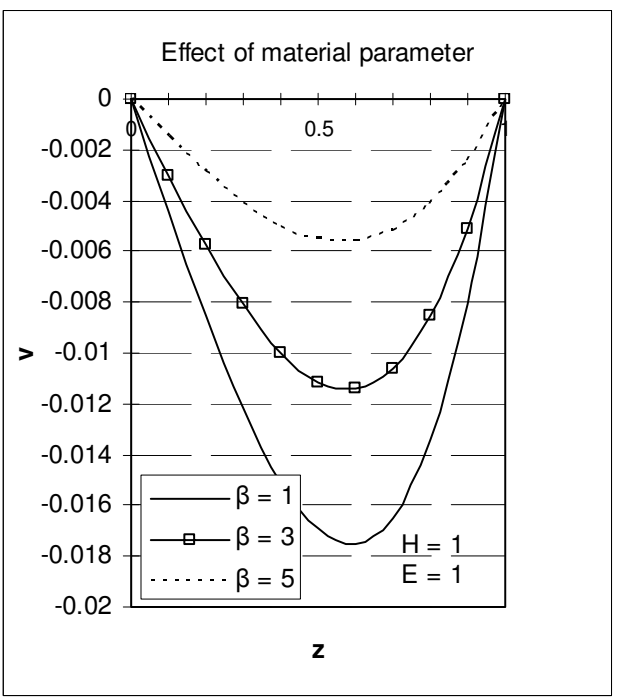

C
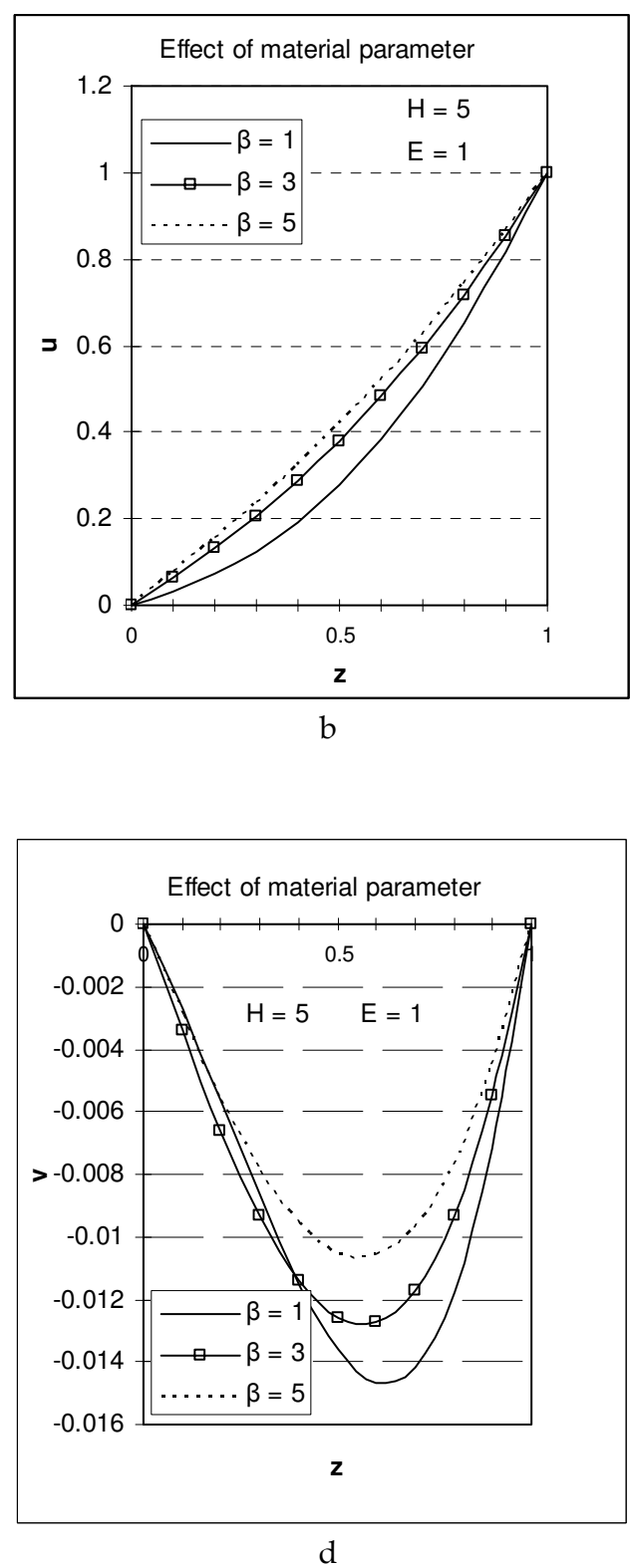

Fig. 3. Variation of velocity components $u$ and $v$ with $z$ for $\beta=1,3$, 5; fixing $E=1, H=5$. 
linear distribution; thus, the shearing can unattenuately extend to the whole flow domain from the boundaries, corresponding to a shear-thickening phenomenon. A further increase of $\beta$ will not effect this velocity component further. The magnitude of velocity component $v$ decreases when $\beta$ increases and the curvature of the velocity profile decreases with an increase in material parameter $\beta$ (see Figs. $3 c, 3 d$ and $4 b$ ). It is also found that the flow behaviour depends strongly on the choice of the parameters, for example, for large $H, u$ increases with an increase of material parameter $\beta$, whereas this velocity component is independent of $\beta$ for small $H$. On the contrary, the magnitude of velocity component $v$ decreases with $\beta$ for both small and large values of $H$.

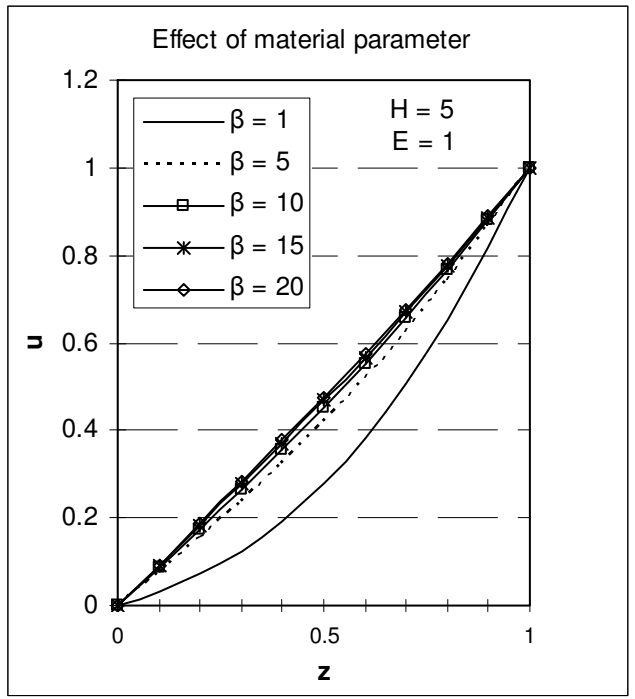

(a)

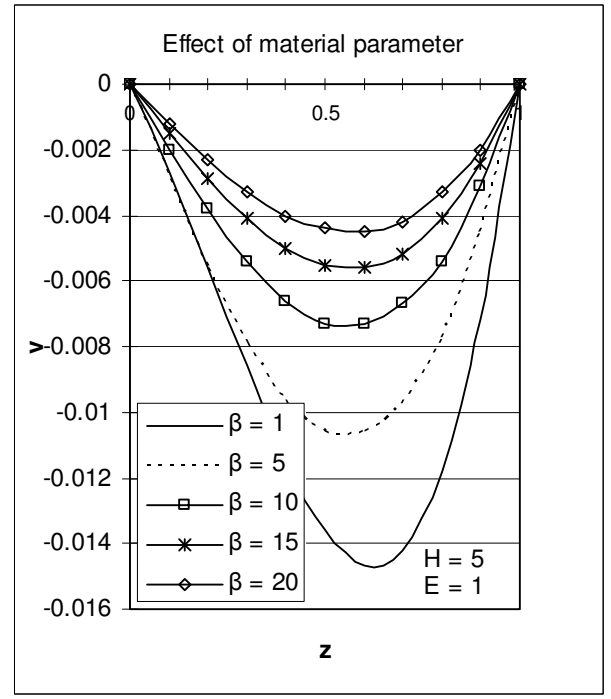

(b)

Fig. 4. Variation of velocity components $u$ and $v$ with $z$ for large values of $\beta=1,5,10,15,20$; fixing $E=1, H=5$.

\section{Conclusions}

The unsteady rotating flow of a uniformly conducting incompressible fourth-grade fluid between two parallel infinite plates in the presence of a magnetic field is modeled. The steady rotating flow of the non-Newtonian fluid subject to a uniform transverse magnetic field is studied. The governing non-linear equations are solved numerically. The numerical results of the non-Newtonian fluid are compared with those of a Newtonian fluid. The major findings of the present works can be summarized as follows:

- The transverse magnetic field decelerates the fluid motion. When the strength of the magnetic field increases, the flow velocity decreases.

- It is observed that the boundary layer thickness decreases drastically by increasing $H$. It means that the magnetic field provides some mechanism to control the boundary layer thickness. 
- It is noted that the flow behaviour depends strongly on the choice of the parameters.

\section{References}

[1] R.S. Rivlin, J.L. Ericksen, Stress deformation relations for isotropic material, J. Rational Mech. Anal. 4 (1955) 323-425.

[2] C. Truesdell and W. Noll, The Nonlinear Field Theories of Mechanics, 2nd ed. SpringerVerlog, Brelin, 1992.

[3] J. G. Oldroyd, On the formulation of rheological equations of state, Proc. Roy. Soc. Lond. A 200 (1950) 523-541.

[4] K.R. Rajagopal, Mechanics of non-Newtonian fluids in Recent Developments in Theoretical Fluid Mechanics, Pitman Res. Notes Math. Ser. 291, eds. G.P. Galdi and J. Necas (Springer), 1993.

[5] J.E. Dunn, K.R. Rajagopal, Fluids of differential type: critical review and thermodynamic analysis, Int. J. Engng. Sci. 33 (1995) 689-729.

[6] R.R. Huilgol, Continuum mechanics of viscoelastic liquids, Hindushan Publishing Corporation, 1975.

[7] W.R. Schowalter, Mechanics of non-Newtonian fluids, Pergamon, 1978.

[8] M.A. Rana, A.M. Siddiqui, Rashid Qamar, Hall effects on hydromagnetic flow of an Oldroyd 6-constant fluid between concentric cylinders, Chaos, Solitons and Fractals 39 (2009) 204-213.

[9] R. Bandelli, K. R. Rajagopal and G.P. Galdi, On some unsteady motions of fluids of second grade. Arch. Mech., 47 (1995) 661 -676.

[10] R.L. Fosdick, K.R. Rajagopal, Anomalous features in the model of "second order fluids", Arch. Ration. Mech. Anal. 70 (1979) 145-152.

[11] R.L. Fosdick, K.R. Rajagopal, Thermaodynamics and stability of fluids of third grade, Proc. Roy. Soc. London Ser. A 369 (1980), no. 1738, 351-377.

[12] J. Malek, K.R. Rajagopal, and M. Ruzicka, Existence and regularity of solutions and stability of the rest state for the fluids with shear dependent viscosity, Math. Models Methods Appl. Sci. 5 (1995), no. 6, 789-812.

[13] Hartmann Hydrodynamics. 1. theory of the lamina flow of an electrically conductive liquid in a homogeneous magnetic field. Kgi Danske Videnslab, Sleskuh, Mat. Fys. Medd. 15 (1937).

[14] G.W. Sutton and A. Shermann, Engineering magneto-hydrodynamics, McGraw-Hill Co. Inc. 1965.

[15] W.F. Hughes and Y.J. Young, The electromagnetinodynamics of fluids, John Wiley, New York, 1966.

[16] T.G. Cowling, Magnetohydrodynamics, Interscience Publishing, Inc., New York, 1957.

[17] S.I. Pai, Magneto-gas dynamics and plasma dynamics, Springer-Verlag, 1962.

[18] K. R. Rajagopal, T.Y. Na, On Stokes problem for non-Newtonian fluid. Acta Mech. 48 (1983) 233 -239.

[19] F. Mollica, K.R. Rajagopal, Secondary flows due to axial shearing of a third grade fluid between two eccentrically placed cylinders. Int. J. Engng. Sci. 37 (1999) 411 - 429.

[20] A.M. Siddiqui, P.N. Kaloni, Plane steady flows of a third grade fluid. Int. J. Engng. Sci. 25 (2) (1987) $171-188$. 
[21] A.M. Sidiqui, M.A. Rana, Naseer Ahmed, Magnetohydrodynamic flow of a Burgers' fluid in an orthogonal rheometer, Applied Mathematical Modelling, 34(2010) 28812892.

[22] A.M. Sidiqui, M.A. Rana, Naseer Ahmed, Hall effects on flow and heat transfer in the hydromagnetic Burgers Ekman layer, Int. J. Moder. Math. 2(2) (2007) 255-268.

[23] S.R. Kasiviswanathan and A.R. Rao, On exact solutions of unsteady MHD flow between eccentrically rotating disks, Arch, Mech., 39(4) (1987) 411-418.

[24] H.K. Mohanty, Hydromagnetic flow between two rotating disk with non-coincident parallel axes of rotation, Phys., Fluids 15 (1972) 1456-1458.

[25] C.S. Erkman, Lett. Appl. Engng. Sci. 3(1975) 51.

[26] S.N. Murthy, R.K.P. Ram, MHD flow and heat transfer due to eccentric rotation of a porous disk and a fluid at infinity, Int. J. Engng. Sci. 16(1978) 943-949.

[27] P.N. Kaloni and A.M. Siddiqui, A note on the flow of viscoelastic fluid between eccentric disks, J. Non-Newtonian Fluid Mech. 26 (1987). 125-133.

[28] A.M. Siddiqui, T. Haroon and S. Asghar, Unsteady MHD flow of non-Newtonian fluid due to eccentric rotations of a porous disk and a fluid at infinity, Acta Mech. 147 (2000), 99-109.

[29] A.M. Sidiqui, M.A. Rana, Naseer Ahmed, Effects of Hall current and heat transfer on MHD flow of a Burgers' fluid due to a pull of eccentric rotating disks, Commun. Nonlinear Sci. Numer. Simul. 13(2008) 1554-1570.

[30] G. Palani, I.A. Abbas, Free Convection MHD Flow with Thermal Radiation from an Impulsively-Started Vertical Plate, Nonlinear Analysis: Modelling and Control, 14(1) (2009) 73-84.

[31] M. Farzaneh-Gord, A. A. Joneidi, and B. Haghighi, Investigating the effects of the important parameters on magnetohydrodynamics flow and heat transfer over a stretching sheet, Proceedings of the Institution of Mechanical Engineers, Part E: Journal of Process Mechanical Engineering 2010224 : 1DOI:10.1243/09544089JPME258.

[32] E.R. Maki, D .Kuzma, R.L. Donnelly, B. Kim, Magnetohydrodynamic lubrication flow between parallel plates. J. Fluid Mech. 26 (1966) 537-543.

[33] W.F. Hughes, R.A. Elco, Magnetohydrodynamic lubrication flow between parallel rotating disks. J. Fluid Mech. 13 (1962) 21-32.

[34] D.C. Kuzma, E.R. Maki, R.J. Donnelly, The magnetohydrodynamic squeeze film, J. Tribol 110 (1988) 375-377.

[35] E.A. Hamza, The magnetohydrodynamic squeeze film, J. Fluid Mech. 19 (1964) 395400.

[36] E.A. Hamza, The magnetohydrodynamic effects on a fluid film squeezed between two rotating surfaces, J. Phys. D: Appl. Phys. 24 (1991) 547-554.

[37] S. Bhattacharyya, A. Pal, Unsteady MHD squeezing flow between two parallel rotating discs, Mech. Res. Commun. 24 (1997) 615-623.

[38] Erik Sweet, K. Vajravelu, A. Robert. Van Gorder, I. Pop, Analytical solution for the unsteady MHD flow of a viscous fluid between moving parallel plates, Commun. Nonlinear Sci. Numer. Simulat. 16 (2011) 266-273.

[39] Z. Abbas, T. Javed, M. Sajid, N. Ali, Unsteady MHD flow and heat transfer on a stretching sheet in a rotating fluid, Journal of the Taiwan Institute of Chemical Engineers 41 (2010) 644-650. 
[40] M. Turkylimazoglu, Unsteady MHD flow with variable viscosity: Applications of spectral scheme, Int. J. Thermal Sci. 49 (2010) 563-570.

[41] T. Hayat, C. Fetecaua, M. Sajid, Analytic solution for MHD Transient rotating flow of a second grade fluid in a porous space, Nonlinear Analysis: Real World Applications 9 (2008) 1619 - 1627.

[42] A.C. Eringen, Simple microfluids, Int. J. Eng. Sci. 2 (1964) 203-217.

[43] A.C. Eringen, Theory of micropolar fluids, Math. Mech. 16 (1966) 1-18.

[44] A.C. Eringen, Theory of thermomicropolar fluids, Math. Anal. Appl. 38 (1972) 480- 496.

[45] N.T. Eldabe, E.F. Elshehawey, Elsayed M.E. Elbarbary, Nasser S. Elgazery, Chebyshev finite difference method for MHD flow of a micropolar fluid past a stretching sheet with heat transfer, Applied Mathematics and Computation 160 (2005) 437-450.

[46] T. Sarpakaya, Flow of non-Newtonian fluids in magnetic field, AIChE J 7 (1961) 324328.

[47] M.I. Char, Heat and mass transfer in a hydromagnetic flow of viscoelastic fluid over a stretching sheet, J. Math. Annal Appl. 186 (1994) 674-689.

[48] A. Raptis, Flow of a micropolar fluid past a continuously moving plate by the presence of radiation, Int. J. Heat Mass transfer 41 (1998) 2865-2866.

[49] A. Raptis, C. Perdikis, Viscoelastic flow by the presence of radiation. ZAMM 78 (1998) 277-279.

[50] A. Raptis, C. Pardikis, H.S. Takhar, Effect of thermal radiation on MHD flow, Appl. Math. Comput. 153 (2004) 645-649.

[51] P. G. Siddheshwar, U.S. Mahabaleswar, Effects of radiation and heat source on MHD flow of a visco-elastic liquid and heat transfer over a stretching sheet, Int. J. Nonlinear Mech. 40 (2005) 807-821.

[52] S.K. Khan, Heat transfer in a viscoelastic fluid flow over a stretching surface with heat source/sink, suction/blowing and radiation, Int. J. Heat Mass transfer 49 (2006) 628-639.

[53] M.S. Abel, P.G. Siddheshwar, M. Nandeppanavar Mahantesh, Heat transfer in a viscoelastic boundary layer flow over a stretching sheet with viscous dissipation and non-uniform heat source, Int. J. Heat Mass Transfer 50 (2007) 960-966.

[54] M.S. Abel, M.M. Nandeppanavar, Heat transfer in MHD viscoelastic boundary layer flow over a stretching sheet with non-uniform heat source/sink, Commun. Nonlinear Sci. Numer. Simul. 14 (2009) 2120-2131.

[55] M.M. Nandeppanavar, M.S. Abel, J. Tawade, Heat transfer in a Walter's liquid B fluid over an impermeable stretching sheet with non-uniform heat source/sink and elastic deformation, Commun. Nonlinear Sci. Numer. Simul. 15 (2010) 17911802.

[56] M. Mahantesh, Nandeppanavar, K. Vajravelu, M. Subhas Abel, Heat transfer in MHD viscoelastic boundary layer flow over a stretching sheet with thermal radiation and non-uniform heat source/sink, Commun. Nonlinear Sci. Numer. Simulat. 16 (2011) 3578-3590.

[57] Kayvan Sadeghy, Navid Khabazi, Seyed-Mohammad Taghavi, Magnetohydrodynamic (MHD) flows of viscoelastic fluids in converging/diverging Channels, Int. J. Engng. Sci. 45 (2007) 923-938. 
[58] B. D. Coleman and W. Noll, An approximation theorem for functionals with applications continuum mechanics, Arch. Rational Mech. Anal. 6 (1960) 355370.

[59] K.A Hoffmann, S. Chiang, Computational fluid dynamics for engineers, I, 1995, P. 92. 


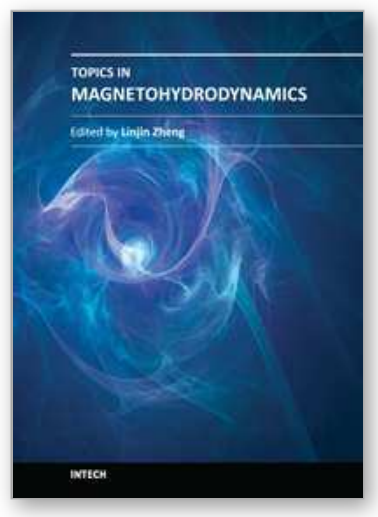

\section{Topics in Magnetohydrodynamics}

Edited by Dr. Linjin Zheng

ISBN 978-953-51-0211-3

Hard cover, 210 pages

Publisher InTech

Published online 09, March, 2012

Published in print edition March, 2012

To understand plasma physics intuitively one need to master the MHD behaviors. As sciences advance, gap between published textbooks and cutting-edge researches gradually develops. Connection from textbook knowledge to up-to-dated research results can often be tough. Review articles can help. This book contains eight topical review papers on MHD. For magnetically confined fusion one can find toroidal MHD theory for tokamaks, magnetic relaxation process in spheromaks, and the formation and stability of field-reversed configuration. In space plasma physics one can get solar spicules and X-ray jets physics, as well as general sub-fluid theory. For numerical methods one can find the implicit numerical methods for resistive MHD and the boundary control formalism. For low temperature plasma physics one can read theory for Newtonian and nonNewtonian fluids etc.

\section{How to reference}

In order to correctly reference this scholarly work, feel free to copy and paste the following:

M.A. Rana, Akhlaq Ahmed and Rashid Qamar (2012). Magnetohydrodynamic Rotating Flow of a Fourth Grade Fluid Between Two Parallel Infinite Plates, Topics in Magnetohydrodynamics, Dr. Linjin Zheng (Ed.), ISBN: 978-953-51-0211-3, InTech, Available from: http://www.intechopen.com/books/topics-inmagnetohydrodynamics/magnetohydrodynamic-rotating-flow-of-a-fourth-grade-fluid-between-two-parallelinfinite-plates

\section{INTECH}

open science | open minds

\section{InTech Europe}

University Campus STeP Ri

Slavka Krautzeka 83/A

51000 Rijeka, Croatia

Phone: +385 (51) 770447

Fax: +385 (51) 686166

www.intechopen.com

\section{InTech China}

Unit 405, Office Block, Hotel Equatorial Shanghai

No.65, Yan An Road (West), Shanghai, 200040, China 中国上海市延安西路65号上海国际贵都大饭店办公楼 405 单元

Phone: +86-21-62489820

Fax: $+86-21-62489821$ 
(C) 2012 The Author(s). Licensee IntechOpen. This is an open access article distributed under the terms of the Creative Commons Attribution 3.0 License, which permits unrestricted use, distribution, and reproduction in any medium, provided the original work is properly cited. 